volume ratio (TVR) ( 0.80 vs. 0.72$)$ were significantly associated with development of edema after SRS $(\mathrm{p}<0.05$, power $>0.8)$. Conclusion: Volume-based reporting of SRS outcomes for meningiomas is more accurate for reporting tumor control. Conformity index and TVR were identified as predictors of edema following radiosurgery.

\section{C4 - Session5 1115-1130}

doi:10.1017/cjn.2014.63

\section{Adjuvant radiosurgery to the tumor bed of resected metastases: A series of 130 patients}

\author{
C Iorio-Morin ${ }^{1}, Y$ Ezahr ${ }^{1}$, L Masson-Cote ${ }^{2}$, J Blanchard ${ }^{1}$, A \\ Ebacher', D Mathieu ${ }^{l}$
}

${ }^{I}$ Department of Neurosurgery, Université de Sherbrooke, Sherbrooke, Quebec: Christian Iorio-Morin, Youssef Ezahr, Jocelyn Blanchard, David Mathieu; ${ }^{2}$ Department of Radiation Oncology, Université de Sherbrooke, Sherbrooke, Quebec: Laurence Masson-Côté, Annie Ebacher

OBJECTIVE Optimal management following surgical removal of brain metastasis remains controversial. To assess the effectiveness and safety profile of tumor bed SRS following surgical resection of brain metastasis, we performed a retrospective analysis of 130 patients who received such treatment at our center. METHODS Patients treated at our center between 2004 and 2013 were included if they had one or more brain metastasis surgically removed, their tumor bed treated by SRS and at least 6 months of available follow-up. Average age at first SRS treatment was 59. At the time of SRS, gross total resection of the brain metastasis had been achieved in $80 \%$ of cases and systemic disease was inactive in $59 \%$ of patients. Tumor bed SRS was performed on average 3.7 weeks following surgery. Mean cavity volume was 12 cc with an average maximal and marginal dose of 36 Gy and 18 Gy respectively. RESULTS Results for the full cohort will be presented at the meeting. Preliminary analysis of 56 of the 130 patients reveals local control at the tumor bed was achieved in $86 \%$ of cases (average follow-up of 13 months). New brain metastases following SRS were identified in $63 \%$ of patients. Median survival was 8 months, with $67 \%$ of patients dying from a systemic rather than neurological cause. CONCLUSION SRS is a safe and effective adjuvant modality following surgical resection of brain metastasis. Pending completion of randomized control trials, our results support the use of SRS for local control of brain disease.

\section{C5 - Session5 1130-1145}

doi:10.1017/cjn.2014.64

\section{Assessing Bimanual Performance in Brain Tumor Resection using a Novel Virtual Reality Simulator NeuroTouch}

F Alotaibi $^{1,2}$, G Al Zhrani ${ }^{1,2}$, H Azarnoush $^{1}$, A Winkler-Schwartz ${ }^{1}$ MMulla, RF Del Maestro ${ }^{l}$

${ }^{I}$ Neurosurgical Simulation Research Center, Montreal Neurological Hospital and Institute, McGill University, Montreal Quebec; ${ }^{2}$ National Neuroscience Institute (NNI), Neurosurgery Department, King Fahad Medical City (KFMC)

Introduction: Validity assessment of NeuroTouch is important in the goal of using it in neurosurgical training, assessment and curriculum development. Methods: This study was conducted to assess bimanual performance of junior, senior resident and consultant resecting simulated brain tumors. Novel metrics were assessed including: total distance travelled by the tip of the ultrasonic aspirator and sucker (TPL), the maximum and sum of forces generated by instruments, blood loss, efficiency and coordination indexes and total brain tissue removed (BTR). Hypotheses: The complexity of tumor will influence neurosurgical performance and this influence will be greater in residents compare to consultants. Novel metrics will differentiate between groups. Results: All groups showed significant difference in 1) the amount of BTR comparing vague to clear boarder 2) simulated ultrasonic aspirator maximum and sum of forces on hard compare to soft tumors. Junior and senior residents showed more differences including 1) significantly more blood loss operating on hard versus soft tumors. 2) Higher ultrasonic aspirator TPL when operating on hard versus soft tumors. Junior resident also showed applied more sum of forces by the suction on the hard compare to the soft tumors.

Significant difference between the consultant, senior, and junior residents efficiency index observed $(75.6 \%, 63.4 \%, 60.3 \%$ respectively $\mathrm{P}=0.001)$. Discussion: This study is the first to demonstrate significant differences in neurosurgical performance based on the complexity of tumor. Increasing tumor complexity influenced the junior resident group most was less of an influence on the senior and was least on the consultant group performance.

C6 - Session5 1145-1200

doi:10.1017/cjn.2014.65

Predictors of survival after second surgery for recurrent glioblastoma multiforme tumours

\author{
A Kilian, K Parvez, E. Monsalves, S Larjani, G Klironomos, G \\ Zadeh
}

Arthur and Sonia Labatt Brain Tumour Research Centre, The Hospital for Sick Children, Toronto, Ontario

Background and Purpose: Glioblastoma multiforme (GBM) tumours are the most common brain tumours among adults. Although numerous treatment modalities exist, GBM has a mean recurrence period of less than seven months and a mean survival period of less than fifteen months. The impact of second surgery on recurrence remains unclear, with few definitive studies to date. 